\title{
THE STONE SPACE OF THE SATURATED BOOLEAN ALGEBRAS
}

\author{
BY \\ S. NEGREPONTIS( ${ }^{(1)}$
}

As a consequence of results by B. Jónsson $\left[\mathbf{J}_{1}\right],\left[\mathbf{J}_{2}\right], \mathbf{M}$. Morley and R. Vaught [MV] $\left.{ }^{2}\right)$, for every cardinal number $\kappa$ such that $\kappa=\kappa^{\kappa}\left(=\sum\left\{\kappa^{\mu}: \mu<\kappa\right\}\right)\left({ }^{3}\right)$ there is a unique (up to Boolean isomorphism) Boolean algebra $\mathscr{B}_{\kappa}$ which is $\kappa$-homogeneous, $\kappa$-universal (in short, $\kappa$-saturated) of cardinality $\kappa$. Let $S_{\kappa}$ be the Stone space of $\mathscr{B}_{k}$, i.e., $S_{\kappa}$ is a compact totally disconnected space, whose Boolean algebra of open-and-closed subsets is isomorphic to $\mathscr{B}_{\kappa}$. The object of the present paper is to characterize (up to homeomorphism) the space $S_{\kappa}$ and to study the behavior of some of its subspaces. This work depends heavily on ideas that are contained in the following papers: $\left[\mathbf{R}_{2}\right],[\mathbf{P}],\left[\mathbf{R}_{1}\right],[\mathbf{H R}],[\mathbf{K}]$, as well as on unpublished research undertaken jointly with Professor W. W. Comfort during the summer of 1967, only a portion of which appears in [CN]. We remark that the space $S_{\kappa}$ will have properties similar to the space $\beta \omega \mid \omega$ of nonprincipal ultrafilters on the set $\omega$ of positive integers. Once the basic properties of $S_{\kappa}$ are established, the arguments leading to these results are similar to the familiar arguments concerning $\beta \omega \mid \omega$. Thus, instead of presenting the proofs in a self-contained manner, we have chosen to rely on the references mentioned above. There seem to be two main open problems concerning the spaces $S_{\kappa}$. These are mentioned in $\$ 2$ (end remarks) and $\S 3$ (end remarks) below.

The author acknowledges with pleasure the helpful conversations he had with Dr. Gonzalo Reyes on the contents of this paper.

1. A cardinal number will be an ordinal number which is an initial segment. The cardinality of a set $S$ will be denoted by $|S|$. A Boolean algebra $\mathscr{B}$ is $\kappa$-universal if every Boolean algebra $\mathscr{C},|\mathscr{C}| \leqq \kappa$, can be embedded into $\mathscr{B}$; a Boolean algebra $\mathscr{B}$ is

Received by the editors March 18, 1968 and, in revised form, August 28, 1968.

(1) The author wishes to acknowledge partial support from the Canadian National Research Council (under grant A-4035) during the period in which research for this paper has been undertaken.

${ }^{2}$ ) The fact that Boolean algebras form a Jónsson class, i.e. a class satisfying conditions

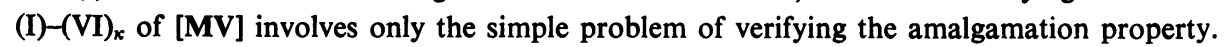
The validity of this property has been noted independently by several authors: Eva Kallin (see last paragraph in [K]), Aubert Daigneault, Ph. Dwinger and F. M. Yaqub [DY], etc.

$\left({ }^{3}\right)$ It is well known that $\kappa=\kappa^{\kappa}$ if and only if $\kappa$ is regular and such that $2^{\alpha} \leqq \kappa$ for all cardinals $\alpha<\kappa$. Thus assuming the generalized continuum hypothesis $\kappa=\kappa^{\kappa}$ if and only if either $\kappa$ is a successor cardinal or $\kappa$ is a strongly inaccessible cardinal (cf. [B $\left.\left.\mathbf{B}_{1}\right]\right)$. 
$\kappa$-homogeneous if given a Boolean algebra $\mathscr{C},|\mathscr{C}|<\kappa$, and two embeddings $\phi, \psi: \mathscr{C} \rightarrow \mathscr{B}$, there is an automorphism $h$ on $\mathscr{B}$ such that $h \circ \phi=\psi$. A Boolean algebra $\mathscr{B}$ is $\kappa$-saturated if it is $\kappa$-homogeneous and $\kappa$-universal. Unless stated otherwise, we assume from now on that $\kappa=\kappa^{\kappa}$, and we identify the unique $\kappa$ saturated Boolean algebra of cardinality $\kappa, \mathscr{B}_{\kappa}$, with the Boolean algebra of its Stone space $S_{\kappa}$. For subsets $\mathscr{A}, \mathscr{B}$ of $\mathscr{B}_{\kappa}$, the notation $\mathscr{A}<\mathscr{B}$ will mean that $\mathscr{A} \subsetneq \mathscr{B}$ for all $A \in \mathscr{A}, B \in \mathscr{B}$. If $\mathscr{A}=\{A\}$ we simply write $A<\mathscr{B}$ etc.

1.1. LeMma. For $\mathscr{A}, \mathscr{B} \subset \mathscr{B}_{k},|\mathscr{A}|+|\mathscr{B}|<\kappa, \mathscr{A}<\mathscr{B}, \mathscr{A}$ directed upwards, $\mathscr{B}$ directed downwards, there is $V \in \mathscr{B}_{x}$, such that $\mathscr{A}<V<\mathscr{B}$, with the following (obvious) exceptions: if $\mathscr{B}=\varnothing, 1 \notin \mathscr{A} ;$ if $\mathscr{A}=\varnothing, 0 \notin \mathscr{B}$.

Proof. Let $S_{1}, S_{2}$ be two copies of $S_{\kappa}$, and $S$ the discrete union of $S_{1}$ and $S_{2}$ (i.e., $S$ is the disjoint union of $S_{1}, S_{2}$, and both $S_{1}, S_{2}$ are open-and-closed in $S$ ). We denote by $\mathscr{B}(S)$ the Boolean algebra of open-and-closed subsets of $S$, by $b(\mathscr{A} \cup \mathscr{B})$ the Boolean subalgebra of $\mathscr{B}_{k}$ generated by $\mathscr{A} \cup \mathscr{B}$. We define an embedding $e$ of $b(\mathscr{A} \cup \mathscr{B})$ into $\mathscr{B}(S)$ by $e(A)=A_{1}$ for $A \in \mathscr{A}, e(B)=S_{1} \cup B_{2}$ for $B \in \mathscr{B}$, and extending the definition of $e$ in the natural way. Indeed, the extension of $e$ can be defined in the following manner. By compactness there is $p \in \cap \mathscr{A} \backslash \cup \mathscr{B}$. Define $e: b(\mathscr{A} \cup \mathscr{B})$ $\rightarrow \mathscr{B}(S)$ by

$$
\begin{aligned}
e(x) & =x_{1} & & \text { if } p \notin x \\
& =S_{1} \cup x_{2} & & \text { if } p \in x\left(^{4}\right) .
\end{aligned}
$$

(Here $A_{1}$ is the copy of $A$ in $S_{1}$, etc.) Let now $\mathscr{C}$ be the Boolean subalgebra of $\mathscr{B}(S)$ generated by $e(\mathscr{A} \cup \mathscr{B})$ and $S_{1}, \mathscr{C}=b\left(e(\mathscr{A} \cup \mathscr{B}) \cup\left\{S_{1}\right\}\right)$. Clearly $|\mathscr{C}|<\kappa$. Let $\phi$ be an embedding of $\mathscr{C}$ into $\mathscr{B}_{x}$. The $\kappa$-homogeneity of $\mathscr{B}_{k}$ now implies the existence of an automorphism $h$ of $\mathscr{B}_{k}$ such that $h \circ(\phi \circ e)=$ inclusion of $b(\mathscr{A} \cup \mathscr{B})$ into $\mathscr{B}_{k}$. The definition $V=h \circ \phi\left(S_{1}\right)$ satisfies the conclusion of the lemma.

1.2. CoRollarY. A maximal totally ordered subset of $\mathscr{B}_{x}$ is an $\eta_{x}$-set (in the sense of Hausdorff) of cardinality $\kappa$ with two endpoints.

1.3. Problem. Assuming the continuum hypothesis $\omega_{1}=2^{\omega}$, it can be shown that for $\kappa=\omega_{1}$ the property of Corollary 1.2 characterizes $\mathscr{B}_{\omega_{1}}$ (cf. [P]). We ask the question: If a Boolean algebra $\mathscr{B}$ of cardinality $\kappa$ satisfies the condition that every maximal totally ordered subset of $\mathscr{B}$ is an $\eta_{k}$-set with two endpoints does it follow that $\mathscr{B}$ is isomorphic to $\mathscr{B}_{\kappa}$ ? (We assume as usual that $\kappa=\kappa \stackrel{\kappa}{*}$.)

1.4. Definition. For an open set $U$ in $S_{x}$, the type of $U, \tau(U)$ is the smallest cardinal number $\alpha$, such that $U$ can be expressed as the union of $\alpha$ open-and-closed subsets of $S_{k}$ (cf. [ $\left.\mathbf{N}_{2}\right]$ ).

1.5. Corollary. Let $U, V$ be open subsets of $S_{x}$.

$\left.{ }^{4}\right)$ This sentence was supplied by the referee. 
(i) If $U$ is proper and $\tau(U)<\kappa$, then $U$ is not dense in $S_{x}$.

(ii) If $\tau(U)<\kappa, \tau(V)<\kappa$, and $U \cap V=\varnothing$, then $\bar{U} \cap \bar{V}=\varnothing$. ( $\bar{U}$ denotes the closure of $U$ in $S_{k}$.)

(iii) If $\tau(U)<\kappa$ then $U$ is $C^{*}$-embedded in $S_{\kappa}$ (in the sense of [GJ]).

(iv) If $1<\tau(U)<\kappa$, then $\bar{U}$ is not an open subset of $S_{\kappa}$; in particular $S_{\kappa}$ is not basically disconnected (in the sense of [GJ]).

Proof. Let $U$ be the union of $\left\{V_{i}: i \in I\right\}$ and $V$ be the union of $\left\{W_{j}: j \in J\right\}$, where $V_{i}, W_{j} \in \mathscr{B}_{\kappa}$ for all $i, j$ and $|I|<\kappa,|J|<\kappa$. For (i) let $\mathscr{A}=\left\{V_{i}: i \in I\right\}, \mathscr{B}=\left\{S_{x}\right\}$. For (ii) let $\mathscr{A}=\left\{V_{i}: i \in I\right\}, \mathscr{B}=\left\{S_{x} \mid W_{j}: j \in J\right\}$. To prove (iv) assume that $\bar{U}$ is open, hence $\bar{U} \in \mathscr{B}_{x}$, and let $\mathscr{A}=\left\{V_{i}: i \in I\right\}$ and $\mathscr{B}=\{\bar{U}\}$. (In fact (i) is a special case of (iv).) For (iii) we use Urysohn's extension theorem, in the general form that appears as Theorem 1.17 in [GJ]. Let $A, B$ be two completely separated subsets in $U$, and let $f$ be a continuous function of $U$ into the interval $[0,1]$, such that $f=0$ on $A$ and $f=1$ on $B$. Let $\tilde{A}=\left\{p \in U: f(p)<\frac{1}{2}\right\}, \widetilde{B}=\left\{p \in U: f(p)>\frac{1}{2}\right\}$. It is clear that $\tilde{A}, \widetilde{B}$ are disjoint open subsets of $S_{\kappa}$, with $\tau(\widetilde{A}) \leqq \tau(U) \cdot \aleph_{0}<\kappa, \tau(\widetilde{B}) \leqq \tau(U) \cdot \aleph_{0}<\kappa$. By (ii), $\tilde{A}, \widetilde{B}$ have disjoint closures in $S_{\kappa}$, and since $S_{\kappa}$ is compact, they are completely separated in $S_{\kappa}$. Thus, an application of Urysohn's extension theorem yields (iii).

Our next lemma is a direct generalization of similar lemmas that have been proved for the case $\kappa=\omega_{1}$ by W. Rudin $\left[\mathbf{R}_{2}\right]$, Parovičenko [P], and Keisler $[\mathbf{K}]$. The proof presented below requires only slight effort over the proof of the lemma for $\kappa=\omega_{1}$ as it has been worked jointly with Professor W. W. Comfort (unpublished).

1.6. LeMma. Let $\mathscr{D}$ be a Boolean algebra satisfying Lemma 1.1 above (i.e. for $\mathscr{A}, \mathscr{B} \subset \mathscr{D},|\mathscr{A}|+|\mathscr{B}|<\kappa, \mathscr{A}<\mathscr{B}$, there is $V \in \mathscr{D}$, such that $\mathscr{A}<V<\mathscr{B}$, with the two obvious exceptions mentioned there). Then $\mathscr{D}$ satisfies the following (stronger) condition: for $\mathscr{A}, \mathscr{B}, \mathscr{C} \subset \mathscr{D},|\mathscr{A}|+|\mathscr{B}|+|\mathscr{C}|<\kappa$, such that

(i) $\mathscr{A}<\mathscr{B}, \mathscr{A}$ directed upwards, $\mathscr{B}$ directed downwards,

(ii) for all $A \in \mathscr{A}, B \in \mathscr{B}, C \in \mathscr{C}, C \leqq A$ and $B \leqq C$ are both false, there is $V \in \mathscr{D}$, such that

(iii) $\mathscr{A}<V<\mathscr{B}$,

(iv) for all $C \in \mathscr{C}, V$ is not comparable to $C$ (i.e. $V \leqq C, C \leqq V$ are both false).

(Of course, we must make again the two obvious exceptions.)

Proof. We let $S$ be the Stone space of $\mathscr{D}$, and we identify $\mathscr{D}$ with the Boolean algebra $\mathscr{B}(S)$ of open-and-closed subsets of $S$. Thus the order $\leqq$ becomes set inclusion. Let $\lambda=|\mathscr{A}|+|\mathscr{B}|+|\mathscr{C}|$. With possible repetitions we well-order $\mathscr{A}$ $=\left\{A_{\alpha}\right\}_{\alpha<\lambda}, \mathscr{B}=\left\{B_{\alpha}\right\}_{\alpha<\lambda}, \mathscr{C}=\left\{C_{\alpha}\right\}_{\alpha<\lambda}$.

We will find nonempty open-and-closed sets $F_{\alpha}, G_{\alpha}, \alpha<\lambda$, such that

$$
\begin{gathered}
F_{\alpha} \subset C_{\alpha} \mid \bigcup_{\beta<\lambda} A_{\beta}, \\
G_{\alpha} \subset \bigcap_{\beta<\lambda} B_{\beta} \mid C_{\alpha}, \quad \text { and } \\
F_{\alpha} \cap G_{\beta}=\varnothing \quad \text { for all } \alpha, \beta<\lambda .
\end{gathered}
$$


We proceed by transfinite induction. Let $F_{1}^{(1)}$ be any nonempty open-and-closed subset of $C_{1} \mid \bigcup_{\beta<\lambda} A_{\beta}$. Notice that this choice is possible, since by 1.5 (i) (which applies to $\mathscr{D}$ as well as to $\left.\mathscr{B}_{\kappa}\right)$ the set $\bigcap_{\beta<\lambda}\left(C_{1} \mid A_{\beta}\right)$ has nonempty interior. In a similar way, let $G_{1}^{(1)}$ be a nonempty open-and-closed subset of $\bigcap_{\beta<\lambda} B_{\beta} \mid C_{1}$. Notice that $F_{1}^{(1)} \cap G_{1}^{(1)}=\varnothing$. Let $\alpha<\lambda$. Suppose that for all $\gamma<\alpha$, we have defined nonempty open-and-closed sets $F_{\delta}^{(r)}, G_{\varepsilon}^{(\gamma)}$, for all $\delta, \varepsilon \leqq \gamma$, such that

$$
\begin{aligned}
& F_{\delta}^{(\delta)} \supset F_{\delta}^{(\delta+1)} \supset \ldots \supset F_{\delta}^{(\gamma)} \supset \ldots, \quad G_{\varepsilon}^{(\varepsilon)} \supset G_{\varepsilon}^{(\varepsilon+1)} \supset \ldots \supset G_{\varepsilon}^{(\gamma)} \supset \ldots, \\
& F_{\delta}^{(\gamma)} \cap G_{\varepsilon}^{(\gamma)}=\varnothing, \\
& F_{y}^{(\gamma)} \subset C_{\gamma}\left|\bigcup_{\beta<\lambda} A_{\beta}, \quad G_{\gamma}^{(\gamma)} \subset \bigcap_{\beta<\lambda} B_{\beta}\right| C_{\gamma} .
\end{aligned}
$$

Let $E_{\gamma}^{(\alpha)}$ be a nonempty open-and-closed subset of $\bigcap_{\delta<\alpha} F_{\gamma}^{(\delta)}$, and let $H_{\gamma}^{(\alpha)}$ be a nonempty open-and-closed subset of $\bigcap_{\delta<\alpha} G_{\gamma}^{(\delta)}$; the choices are possible by 1.5 (i). Notice that if $\alpha=\beta+1$, we may simply take $E_{\gamma}^{(\alpha)}=F_{\gamma}^{(\beta)}, H_{\gamma}^{(\alpha)}=G_{\gamma}^{(\beta)}$. At this point we employ a "subsidiary" transfinite induction (the details will be omitted) to define $F_{\alpha}^{(\alpha)}$ to be any nonempty open-and-closed subset of $C_{\alpha} \mid \bigcup_{\beta<\lambda} A_{\beta}$, such that $H_{\gamma}^{(\alpha)} \backslash F_{\alpha}^{(\alpha)}$ $\neq \varnothing$ for all $\gamma<\alpha$, and $G_{\alpha}^{(\alpha)}$ to be a nonempty open-and-closed subset of $\bigcap_{\beta<\lambda} B_{\beta} \mid C_{\alpha}$, such that $E_{\gamma}^{(\alpha)} \mid G_{\alpha}^{(\alpha)} \neq \varnothing$ for all $\gamma<\alpha$. We then set $G_{\gamma}^{(\alpha)}=H_{\gamma}^{(\alpha)}\left|F_{\alpha}^{(\alpha)}, F_{\gamma}^{(\alpha)}=E_{\gamma}^{(\alpha)}\right| G_{\alpha}^{(\alpha)}$ for all $\gamma<\alpha$. It can be seen that conditions (1), (2), (3) above are satisfied for $\alpha$. Finally we let $F_{\alpha}, G_{\alpha}$ be any nonempty open-and-closed subsets of $\bigcap_{\delta<\lambda} F_{\alpha}^{(\delta)}, \bigcap_{\delta<\lambda} G_{\alpha}^{(\delta)}$, respectively, using again $1.5(\mathrm{i})$.

Let now $\tilde{A}=\left\{A_{\alpha_{1}} \cup G_{\alpha_{1}} \cup \ldots \cup A_{\alpha_{n}} \cup G_{\alpha_{n}}: \alpha_{1}, \ldots, \alpha_{n}<\lambda, n<\omega\right\}$,

$$
\tilde{\mathscr{B}}=\left\{\left(B_{\alpha_{1}} \mid F_{\alpha_{1}}\right) \cap \cdots \cap\left(B_{\alpha_{n}} \mid F_{\alpha_{n}}\right): \alpha_{1}, \ldots, \alpha_{n}<\lambda, n<\omega\right\} ;
$$

it is clear that $\tilde{\mathscr{A}}<\tilde{\mathscr{B}}, \tilde{\mathscr{A}}$ directer upwards, $\tilde{\mathscr{B}}$ directed downwards, and thus there is a nonempty open-and-closed set $V$, such that $\tilde{\mathscr{A}}<V<\tilde{\mathscr{B}}$; the set $V$ satisfies the conditions of the lemma.

The following result provides a topological characterization of the Stone space $S_{x}$.

1.7. THEOREM. The space $S_{\kappa}$ is a compact, totally disconnected space, having $\kappa$ open-and-closed sets, and with no isolated points, satisfying the following condition:

Every proper open set of type less than $\kappa$ is not dense and it is $C^{*}$-embedded.

Moreover, any two spaces satisfying these conditions are homeomorphic.

Proof. That $S_{x}$ satisfies the above conditions follows from 1.1, 1.5. The uniqueness follows by a familiar transfinite induction (cf. $\left[\mathbf{R}_{2}\right],[\mathbf{P}],[\mathbf{H R}],[\mathbf{K}]$ ) using Lemma 1.6, and will be omitted.

A partial order $\geqq$ on a set $P$ will be assumed to satisfy in addition to the axioms of reflexivity and transitivity, the antisymmetric axiom: $x \geqq y, y \geqq x$ implies $x=y$. It was shown in $\left[J_{1}\right]$ that the system of partially ordered sets is a Jónsson class (i.e. it satisfies (I)-(VI) $)_{\kappa}$ in [MV]) and, hence, admits saturated structures. 
1.8. THEOREM. As a partially ordered set $\mathscr{B}_{\kappa}$ is $\kappa$-universal.

The proof follows from 1.6, the fact that every partially ordered set can be embedded in a directed partially ordered set, and applications of well-known arguments and, thus, will be omitted.

We remark, however, that as a partially ordered set, $\mathscr{B}_{\kappa}$ is not $\kappa$-homogeneous, since it is clear that the $\kappa$-saturated partially ordered set cannot have a first, or last element. Even if we delete the endpoints of $\mathscr{B}_{k}$, the resulting set fails to be the $\kappa$-saturated partially ordered set $\left({ }^{5}\right)$. Indeed, it is easy to verify that a characteristic property of the $\kappa$-saturated partially ordered set $\mathscr{P}_{\kappa}$ is the following: given $\mathscr{A}, \mathscr{B}$, $\mathscr{C} \subset \mathscr{P}_{\kappa}$, such that $|\mathscr{A}|+|\mathscr{B}|+|\mathscr{C}|<\kappa, \mathscr{A}<\mathscr{B}$, and the relations $a \leqq c, c \leqq b$ are false for all $a \in \mathscr{A}, b \in \mathscr{B}, c \in \mathscr{C}$, then there is $v \in \mathscr{P}_{k}$, such that $\mathscr{A}<v<\mathscr{B}$, and such that the relations $v \leqq c, c \leqq v$ are false for all $c \in \mathscr{C}$. It is to be noted that $\mathscr{B}_{\boldsymbol{x}}$ satisfies the above property, according to Lemma 1.6 above, only for sets $\mathscr{A}, \mathscr{B}, \mathscr{C}$ as above, that in addition have $\mathscr{A}$ directed upwards and $\mathscr{B}$ directed downwards.

2. By Stone's duality between Boolean algebras and totally disconnected compact spaces (in a categorical sense) the following statements are true for $S_{x}$ :

(a) Every compact totally disconnected space having at most $\kappa$ open-and-closed sets is the continuous epimorphic image of $S_{\kappa}$.

(b) For any compact totally disconnected space $K$, with less than $\kappa$ open-andclosed sets, and any two continuous epimorphisms $f, g$ of $S_{x}$ onto $K$, there is an autohomeomorphism $h$ of $S_{x}$, such that $g \circ h=f$.

We improve these statements by omitting the restriction that $K$ is totally disconnected, with the aid of the following simple lemmas. For a topological space $K$, the weight $w(K)$ of $K$ is the smallest cardinal number $\alpha$, such that $K$ has a base for its topology of cardinality $\alpha$.

2.1. LEMMA. Let $f$ be an epimorphism of the compact totally disconnected space $S$ onto the compact space $K$. There is a compact totally disconnected space $E$, with $w(K)$ open-and-closed subsets, and epimorphisms $g: S \rightarrow E, h: E \rightarrow K$, such that $h \circ g=f$.

Proof. We may assume that $w(K)$ is infinite. Let $\mathscr{A}$ be a base for the topology, consisting of cozero-sets (cf. [GJ]) and having cardinality $w(K)$. For $A \in \mathscr{A}$, it is easy to find a sequence of open-and-closed subsets of $S, V_{i, A}, i \in \omega$, such that $f^{-1}(A)=\bigcup\left\{V_{i, A}: i \in \omega\right\}$. Let $\mathscr{B}$ be the Boolean subalgebra of open-and-closed subsets of $S$, generated by all sets of the form $V_{i, A}$ for $A \in \mathscr{A}, i \in \omega$, and let $E$ be the Stone space of $\mathscr{B}$. Since $\mathscr{B}$ is a subalgebra of the Boolean algebra of $S$, there is an epimorphism $g: S \rightarrow E$ induced by the inclusion of algebras. By the definition of $E$,

$\left(^{5}\right)$ The fact that $\mathscr{B}_{\kappa}$ is not $\kappa$-homogeneous as a partially ordered set was mentioned to the author by the referee. The fact that $\mathscr{B}_{\kappa}$, even with its endpoints removed, is not $\kappa$-homogeneous as a partially ordered set was remarked to the author by Professor M. Morley. 
it is clear that for every $e \in E$, the function $f$ is constant on $g^{-1}(e)$. Let $h: E \rightarrow K$ be given by $h(e)=f\left(g^{-1}(e)\right)$. Since $g$ is a quotient mapping, $h$ is continuous. It is clear that $|\mathscr{B}|=|\mathscr{A}|=w(K)$.

The second lemma is related to the amalgamation property for Boolean algebras (cf. [DY]).

2.2. Lemma. Given compact spaces $E_{1}, E_{2}, K$ and continuous epimorphisms $h_{1}: E_{1} \rightarrow K, h_{2}: E_{2} \rightarrow K$, there is a compact space $E$, such that $w(E)=w\left(E_{1}\right)+w\left(E_{2}\right)$, and continuous epimorphisms $k_{1}: E \rightarrow E_{1}, k_{2}: E \rightarrow E_{2}$, such that $h_{1} \circ k_{1}=h_{2} \circ k_{2}$. Moreover, if $E_{1}, E_{2}$ are totally disconnected, so is $E$.

Proof. Let $E=\left\{(x, y) \in E_{1} \times E_{2}: h_{1}(x)=h_{2}(y)\right\}$. It is clear that $E$ is compact, and that it is totally disconnected if $E_{1}, E_{2}$ are totally disconnected. Let $k_{1}, k_{2}$ be the restrictions to $E$ of the usual projections of $E_{1} \times E_{2}$ onto $E_{1}, E_{2}$ respectively. Since $h_{1}, h_{2}$ are epimorphic, it is clear that $k_{1}, k_{2}$ are epimorphic. Thus $w(E)=w\left(E_{1}\right)$ $+w\left(E_{2}\right)$, and $h_{1} \circ k_{1}=h_{2} \circ k_{2}$.

The next lemma is a special case of Corollary 2.4 (d) in [MV] which we state here for reference.

2.3. LEMMA. Given compact totally disconnected spaces $E, E_{1}$, each with less than $\kappa$ open-and-closed sets, and continuous epimorphisms $g: S_{\kappa} \rightarrow E_{1}, k: E \rightarrow E_{1}$, there is a continuous epimorphism $l: S_{k} \rightarrow E$, such that $k \circ l=g$.

We now prove the statement corresponding to (b) above, and which may be called (if consistency is required) the $\kappa$-co-homogeneity of $S_{\kappa}$ for compact spaces.

2.4. THEOREM. Whenever $K$ is a compact space of weight less than $\kappa$, and $f, g$ are continuous epimorphisms of $S_{k}$ onto $K$, there is an autohomeomorphism $h$ on $S_{k}$ such that $g \circ h=f$.

Proof. We will refer to the following diagram:

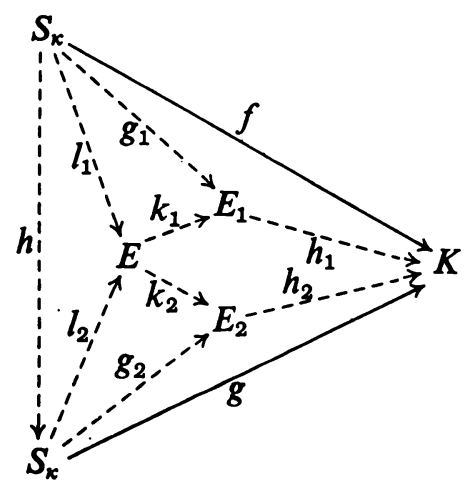

By Lemma 2.1, there are compact totally disconnected spaces $E_{1}, E_{2}$, with $w(K)$ open-and-closed sets, and epimorphisms $g_{1}: S_{\kappa} \rightarrow E_{1}, h_{1}: E_{1} \rightarrow K, g_{2}: S_{\kappa} \rightarrow E_{2}$, $h_{2}: E_{2} \rightarrow K$ such that $h_{1} \circ g_{1}=f, h_{2} \circ g_{2}=g$. By 2.2 , there is a compact totally dis- 
connected space $E$, with $w(K)$ open-and-closed sets, and epimorphisms $k_{1}: E \rightarrow E_{1}$, $k_{2}: E \rightarrow E_{2}$, such that $h_{1} \circ k_{1}=h_{2} \circ k_{2}$. By 2.3, there are epimorphisms $l_{1}, l_{2}: S_{\kappa} \rightarrow E$ such that $k_{1} \circ l_{1}=g_{1}, k_{2} \circ l_{2}=g_{2}$. Finally, by property (b) above, since $w(K)<\kappa$, there is an autohomeomorphism $h$ of $S_{\kappa}$, such that $l_{2} \circ h=l_{1}$. Thus $g \circ h=h_{2} \circ g_{2} \circ h=\left(h_{2} \circ k_{2}\right) \circ\left(l_{2} \circ h\right)=h_{1} \circ k_{1} \circ l_{1}=h_{1} \circ \dot{g}_{1}=f$.

2.5. Lemma. For any compact space $K$, there is a compact totally disconnected space $S$, with $w(K)$ open-and-closed sets, and a continuous epimorphism $h$ of $S$ onto $K$.

Proof. Let $\phi$ be a continuous epimorphism of the Cantor set $C$ onto the unit interval $I$, and $\Phi$ the induced continuous epimorphism of $C^{w(K)}$ onto $I^{w(K)}$. It is clear that $K$ may be considered as a closed subset of $I^{w(K)}$. Let $S$ be the preimage, under $\Phi$, of $K$, and $h=\Phi \mid S$.

The resulting statement is the analogue of (a) above and may be called the $\kappa$-co-universality of $S_{\kappa}$ for compact spaces.

2.6. THEOREM. Every compact space of weight at most $\kappa$ is the continuous epimorphic image of $S_{\kappa}$.

For the proof we use 2.5 and (a).

For completeness we state the following consequence of [MV], [DY] and 2.4, 2.6 above, which provides a second description of $S_{\kappa}$.

2.7. THEOREM. $S_{\kappa}$ is the unique (up to homeomorphism) compact space of weight $\kappa$, which is $\kappa$-co-homogeneous and $\kappa$-co-universal.

By Keisler [K], $S_{\omega_{1}}$ can be identified with $\beta \omega \mid \omega$ (under the continuum hypothesis $\left.\omega_{1}=2^{\omega}\right)$. As a particular case of $2.7, \beta \omega \mid \omega$ can be characterized uniquely up to homeomorphism by the following properties (assuming the continuum hypothesis):

(a) $\beta \omega \mid \omega$ is a compact space of weight $\omega_{1}$,

(b) every compact metric space is the continuous epimorphic image of $\beta \omega \mid \omega$, and

(c) $\beta \omega \mid \omega$ is projective in the category of compact metric spaces and continuous epimorphisms in the following sense: given compact metric spaces $X, Y$, and continuous epimorphisms $g: \beta \omega \mid \omega \rightarrow Y, f: X \rightarrow Y$, there is a continuous epimorphism $h: \beta \omega \mid \omega \rightarrow X$, such that $f \circ h=g$.

Esenin-Vol'pin [E] assuming the generalized continuum hypothesis has proved the existence, for every cardinal $\kappa$ (not necessarily $\kappa=\kappa^{\kappa}$ ), of a compact space of weight $\kappa$, which is, in the above sense, $\kappa$-co-universal for compact spaces. Assume the generalized continuum hypothesis, and let $\kappa$ be any cardinal number. If $\kappa$ is regular (in particular if $\kappa=\alpha^{+}$for some $\alpha$ ) it follows from Theorem 2.7 that, in fact, $S_{\kappa}$ is up to homeomorphism the only $\kappa$-co-homogeneous and $\kappa$-co-universal compact space of weight $\kappa$.

If $\kappa$ is singular the dual notion of $\kappa$-special of Morley and Vaught [MV] may be added to $\kappa$-co-universality to produce a unique compact space. 
As it is remarked in [MV] the results of Sierpiński $\left[\mathbf{S}_{1}\right]$ concerning universal metric spaces can be improved in a similar way. Since compact spaces are related in a dual way both to Boolean algebras and to Banach spaces, and since, according to $[\mathbf{K}]$ (and implicitly according to $\left[\mathbf{R}_{2}\right],[\mathbf{P}],[\mathbf{H R}]$ ) the space $\beta \omega \mid \omega$ is the $\omega_{1}$-couniversal, $\omega_{1}$-co-homogeneous compact space of cardinality $\omega_{1}$, it is natural to ask whether the Banach space $C(\beta \omega \mid \omega)$ of continuous bounded real-valued functions on $\beta \omega \mid \omega$ is the $\omega_{1}$-saturated metric space of cardinality $\omega_{1}$. At least the property of $\omega_{1}$-homogeneity fails according to an obvious modification of a simple idea of Sierpiński $\left[\mathbf{S}_{1}\right]$. It is, in fact, not known to the author whether the $\kappa$-saturated metric spaces of cardinality $\kappa$ have the structure of a Banach space. But the idea of Sierpiński is sufficient to show that they cannot have the form $C(S)$ for a compact space $S$.

The following problem is open: let $\kappa=\alpha^{+}=2^{\alpha}$. Is it possible to represent $S_{\kappa}$ as a space of the form $\beta X_{\kappa} \mid X_{\kappa}$, where $X_{\kappa}$ is a locally compact, realcompact (or even paracompact) space, which can be expressed as the union of (at most) $\alpha$ compact spaces? For $\kappa=\omega_{1}$, we simply take $X_{\kappa}=\omega$. Unfortunately, as it has been remarked by Keisler [K], and as it also follows from [FG], [ $\left.\mathbf{N}_{2}\right]$, the choice $X_{\kappa}=\alpha$ does not work. In fact, even the space of uniform ultrafilters on $\alpha$ is not suitable. A possible choice is to take $X_{\kappa}$ as an open subset of $S_{\kappa}$ of type $\alpha$, e.g. the pairwise disjoint union of $\alpha$ open-and-closed nonempty subsets of $S_{\kappa}$. As it may be seen from [CN] this is suitable for $\kappa=\omega_{1}$.

3. Open subspace of $S_{\kappa}$. Let $U$ be an open subset of $S_{\kappa}$. If $\tau(U)<\kappa$, by 1.5 (iv), $U$ is $C^{*}$-embedded in $S_{\kappa}$. Our initial result below will establish a much stronger property for such open sets $U$, i.e. that for any compact space $K$, and any continuous mapping $f: U \rightarrow K$, there is a continuous extension $g: S_{\kappa} \rightarrow K$ of $f$. As a consequence $\bar{U}$ will be a retract of $S_{\kappa}$. In contrast, if $\tau(U)=\kappa$, there is a two-valued continuous function on $U$ which cannot be continuously extended to $S_{\kappa}$ (cf. [FG]).

3.1. THEOREM. Let $U$ be an open subspace of $S_{\kappa}, \tau(U)<\kappa$, and let $f$ be a continuous function of $U$ into any compact space $K$. There is a continuous extension $g$ of $f$, $g: S_{k} \rightarrow K$.

Proof. Since $U$ is $C^{*}$-embedded in $S_{\kappa}, \bar{U}=\beta U$, and $\bar{U}$ has at most $\kappa$ open-andclosed sets. It is clear, therefore, that we may assume that the weight of $K$ is at most $\kappa$ and that $f$ maps $U$ onto a dense subspace of $K$. We first prove the result for the case that $K$ is totally disconnected. The Boolean algebra $\mathscr{C}$ of open-and-closed sets of $K$ has cardinality at most $\kappa$. Let $\mathscr{B}(U)$ be the Boolean algebra of cpen-and-closed subsets of $U$; since $\bar{U}=\beta U$, we may identify $\mathscr{B}(U)$ with $\mathscr{B}(\bar{U})$, and the restriction mapping $R: \mathscr{B}_{\kappa} \rightarrow \mathscr{B}(U)$ is an epimorphism. Further, let $f^{\#}: \mathscr{C} \rightarrow \mathscr{B}(U)$ be the monomorphism given by $f^{\#}(C)=f^{-1}(C)$. We wish to define a monomorphism $e: \mathscr{C} \rightarrow \mathscr{B}_{\kappa}$, such that $R \circ e=f^{\#}$. Let $\left\{C_{\alpha}\right\}_{\alpha<\kappa}$ be a well-ordering of $\mathscr{C}$, with repetitions if necessary, $C_{0}=\varnothing, \mathscr{C}_{\alpha}=$ the Boolean subalgebra of $\mathscr{C}$ generated by $\left\{\mathscr{C}_{\gamma}\right\}_{\gamma \leqq \alpha}, \tilde{\mathscr{C}}_{\alpha}=$ the Boolean subalgebra of $\mathscr{C}$ generated by $\left\{C_{\gamma}\right\}_{\gamma<\alpha}$. Let $e_{0}\left(C_{0}\right)=\varnothing$, 
$e_{0}(K)=S_{\kappa}$. Let $\alpha<\kappa$, and assume that for all $\gamma<\alpha$, we have defined embeddings $e_{\gamma}: \mathscr{C}_{\gamma} \rightarrow \mathscr{B}_{x}$ such that

(i) $e_{\delta} \subset e_{\gamma}$ for $\delta<\gamma<\alpha$,

(ii) $e_{\gamma}(A) \cap U=f^{-1}(A)$ for all $A \in \mathscr{C}_{\gamma}$,

(iii) $e_{\gamma}(A)=f^{-1}(A)$ for all $A \in \mathscr{C}_{\gamma}$ for which $f^{-1}(A) \in \mathscr{B}_{x}$.

Notice that $\tilde{\mathscr{C}}_{\alpha}=\bigcup_{\gamma<\alpha} \mathscr{C}_{\gamma}$, and let $\tilde{e}_{\alpha}=\bigcup_{\gamma<\alpha} e_{\gamma}: \tilde{\mathscr{C}}_{\alpha} \rightarrow \mathscr{B}_{\kappa}$. Clearly $\tilde{e}_{\alpha}$ is an embedding.

Case 1. If $C_{\alpha} \in \tilde{\mathscr{C}}_{\alpha}, \mathscr{C}_{\alpha}=\tilde{\mathscr{C}}_{\alpha}$, we set $e_{\alpha}=\tilde{e}_{\alpha}$.

Suppose now that $C_{\alpha} \notin \tilde{\mathscr{C}}_{\alpha}$. Then $\mathscr{C}_{\alpha}$ is the Boolean subalgebra of $\mathscr{C}$ generated by $\tilde{\mathscr{C}}_{\alpha}$ and $C_{\alpha}$, i.e. $\mathscr{C}_{\alpha}=\left\{\left(A \cap C_{\alpha}\right) \cup\left(B \backslash C_{\alpha}\right): A, B \in \tilde{\mathscr{C}}_{\alpha}\right\}$.

Case 2. There is $\gamma<\alpha$, such that

$$
C_{\gamma} \subset C_{\alpha} \text { and } f^{-1}\left(C_{\alpha}\right) \backslash f^{-1}\left(C_{\gamma}\right) \in \mathscr{B}_{\kappa}
$$

(i.e. $f^{-1}\left(C_{\alpha}\right) \backslash f^{-1}\left(C_{\gamma}\right)$ is compact). Let $e_{\alpha}\left(C_{\alpha}\right)=\left(f^{-1}\left(C_{\alpha}\right) \backslash f^{-1}\left(C_{\gamma}\right)\right) \cup e_{\gamma}\left(C_{\gamma}\right)$, $e_{\alpha}\left(\left(A \cap C_{\alpha}\right) \cup\left(B \backslash C_{\alpha}\right)\right)=\left[\tilde{e}_{\alpha}(A) \cap e_{\alpha}\left(C_{\alpha}\right)\right] \cup\left[\tilde{e}_{\alpha}(B) \backslash e_{\alpha}\left(C_{\alpha}\right)\right]$, for $A, B \in \tilde{\mathscr{C}}_{\alpha}$.

Case 3. There is $\gamma<\alpha$, such that

$$
C_{\alpha} \subset C_{\gamma} \text { and } f^{-1}\left(C_{\gamma}\right) \backslash f^{-1}\left(C_{\alpha}\right) \in \mathscr{B}_{\kappa}
$$

(i.e. $f^{-1}\left(C_{\gamma}\right) \backslash f^{-1}\left(C_{\alpha}\right)$ is compact). Let $e_{\alpha}\left(C_{\alpha}\right)=e_{\gamma}\left(C_{\gamma}\right) \backslash f^{-1}\left(C_{\alpha}\right)$, etc. The verification that in Cases 2 and $3 e_{\alpha}$ is a well-defined embedding, satisfying (i), (ii), (iii) is not difficult.

Case 4. Suppose that the above three cases fail. Notice that $f^{-1}\left(C_{\alpha}\right)$ is an openand-closed subset of $U$ and hence $\tau\left(f^{-1}\left(C_{\alpha}\right)\right)<\kappa, \tau\left(U \mid f^{-1}\left(C_{\alpha}\right)\right)<\kappa$. Let $\left\{V_{i}: i \in I\right\}$, $\left\{W_{j}: j \in J\right\}$ be two families of open-and-closed subsets of $S_{\kappa}$, directed upwards, and such that $|I|+|J|<\kappa, \bigcup_{i \in I} V_{i}=f^{-1}\left(C_{\alpha}\right), \bigcup_{j \in J} W_{j}=U \mid f^{-1}\left(C_{\alpha}\right)$. Set

and

$$
\begin{aligned}
\mathscr{A} & =\left\{e_{\gamma}\left(C_{\gamma}\right) \cup V_{i}: C_{\gamma} \subset C_{\alpha}, \gamma<\alpha, i \in I\right\}, \\
\mathscr{B} & =\left\{e_{\gamma}\left(C_{\gamma}\right) \mid W_{j}: C_{\alpha} \subset C_{\gamma}, \gamma<\alpha, j \in J\right\},
\end{aligned}
$$

$$
\mathscr{C}=\left\{e_{\gamma}\left(C_{\gamma}\right):\left(C_{\gamma} \mid C_{\alpha}\right) \cup\left(C_{\alpha} \mid C_{\gamma}\right) \neq \varnothing, \gamma<\alpha\right\} .
$$

Notice that $|\mathscr{A}|+|\mathscr{B}|+|\mathscr{C}|<\kappa$, that $\mathscr{A}$ is directed upwards, $\mathscr{B}$ is directed downwards, and $\mathscr{A}<\mathscr{B}$, and that for $A \in \mathscr{A}, B \in \mathscr{B}, C \in \mathscr{C}, C \notin A, B \nsubseteq C$. Hence, by Lemma 1.6, there is an open-and-closed set $e_{\alpha}\left(C_{\alpha}\right)$ such that $\mathscr{A}<e_{\alpha}\left(C_{\alpha}\right)<\mathscr{B}$, and $e_{\alpha}\left(C_{\alpha}\right)$ is comparable with no element of $\mathscr{C}$. Notice that $V_{i} \subset e_{\alpha}\left(C_{\alpha}\right) \subset S_{x} \mid W_{j}$ for all $i \in I, j \in J$, and hence $e_{\alpha}\left(C_{\alpha}\right) \cap U=f^{-1}\left(C_{\alpha}\right)$. Define now $e_{\alpha}: \mathscr{C}_{\alpha} \rightarrow \mathscr{B}_{k}$, by

$$
e_{\alpha}\left(\left(A \cap C_{\alpha}\right) \cup\left(B \mid C_{\alpha}\right)\right)=\left[\tilde{e}_{\alpha}(A) \cap e_{\alpha}\left(C_{\alpha}\right)\right] \cup\left[\tilde{e}_{\alpha}(B) \mid e_{\alpha}\left(C_{\alpha}\right)\right] \text { for } A, B \in \tilde{\mathscr{C}}_{\alpha} .
$$

The verification that $e_{\alpha}$ is an embedding satisfying (i), (ii), (iii) requires a lengthy, but routine argument which will be omitted. Finally, we set $e=\bigcup_{\alpha<x} e_{\alpha}: \mathscr{C} \rightarrow \mathscr{B}_{x}$, an embedding of $\mathscr{C}$ into $\mathscr{B}_{x}$ such that $R \circ e=f^{\#}$. Let $g$ be the dual (to $e$ ) continuous epimorphism of $S_{\kappa}$ onto $K$; by Stone's duality for Boolean algebras $g$ is an extension of $f$. 
Consider now the general case with $K$ any compact space. By 2.1 there is a totally disconnected compact space $D$, and epimorphisms $g: \vec{U} \rightarrow D, h: D \rightarrow K$, such that $h \circ g=f^{\beta}=$ the Stone extension of $f$ to $\beta U=\bar{U}$. By our argument above, there is a continuous extension $\bar{g}$ of $g$ over $S_{k}$. The mapping $\bar{f}=h \circ \bar{g}$ provides the required extension of $f$ over $S_{\kappa}$.

3.2. Corollary. Let $F$ be a closed subset of $S_{\kappa}$ containing densely an open subspace $U, \tau(U)<\kappa$. Then there is a retraction of $S_{\kappa}$ onto $F$.

Proof. Apply 3.1 to the inclusion of $U$ into $F$.

For example, the closure of a cozero-set in $\beta \omega \mid \omega$ (or, of $S_{\kappa}$ ) is a retract of $\beta \omega \mid \omega$ (or, of $S_{\kappa}$ ).

The following theorem is proved in a similar way as Theorem 3.1, by a "backand-forth" induction. For $\kappa=\omega_{1}$ cf. Theorem 9 in $\left[\mathbf{R}_{1}\right]$, and the remarks following Theorem 2.1 in $[\mathbf{C N}]$. The details will be omitted.

3.3. THEOREM. Let $U, V$ be proper open subsets of $S_{\kappa}, \tau(U)<\kappa, \tau(V)<\kappa$, and $h$ a homeomorphism of $U$ onto $V$. There is an autohomeomorphism $g$ of $S_{\kappa}$, such that $g \mid U=h$.

3.4. Corollary. There is a space $\Omega_{\kappa}$ expressible in the form $\Omega_{\kappa}=\bigcup_{\alpha<\kappa}\left(S_{\kappa}\right)_{\alpha}$, where each $\left(S_{\kappa}\right)_{\alpha}$ is a homeomorph of $S_{\kappa}$, open-and-closed in $\Omega_{\kappa}$, and such that $\left(S_{\kappa}\right)_{\alpha} \supsetneqq \bigcup_{\gamma<\alpha}\left(S_{\kappa}\right)_{\gamma}$. Furthermore, up to homeomorphism $\Omega_{\kappa}$ is the only such space.

The proof of this statement is an easy application of 3.3. In order to examine some copies of $\Omega_{\kappa}$ in $S_{\kappa}$ we introduce the following definitions. A space is of " $\kappa$ Baire category" if the intersection of at most $\kappa$ open dense subsets is dense in the space. A point $p$ in a space $S$ is a $P_{\kappa}$-point if the intersection of less than $\kappa$ neighborhoods of $p$ is a neighborhood of $p$. The usual $P$-point, as defined in [GJ], is in the above notation a $P_{\omega_{1}}$-point. As it has been proved by W. Rudin $\left[\mathbf{R}_{2}\right], \beta \omega \mid \omega$ has a dense set of $2^{c} P_{\omega_{1}}$-points. As it has been remarked by Parovičenko [P], $\beta \omega \mid \omega$ is of $\omega_{1}$ Baire category. For $\kappa=\omega_{1}$, the fact that $\beta \omega \mid \omega$ is not point-homogeneous has been recently proved by $Z$. Frolík [F], without any use of the continuum hypothesis. His proof does not establish however the existence of $P$-points in $\beta \omega \mid \omega$. It has been announced by D. D. Booth $\left[\mathbf{B}_{2}\right]$ that the existence of $P$-points in $\beta \omega \mid \omega$ is consistent with certain denials of the continuum hypothesis.

3.5. TheOREM. Let $\kappa>\omega . S_{\kappa}$ is of $\kappa$ Baire category, and it contains a dense set of $2^{\kappa} P_{x}$-points. In particular, $S_{\kappa}$ is not point-homogeneous.

The proof of the first statement can be easily given, using 1.5. For the existence of $P_{\kappa}$-points, notice that a point $p \in S_{\kappa}$ is a $P_{\kappa}$-point if and only if $p$ does not belong to the boundary of an open set $U, \tau(U)<\kappa$. Thus the set $P_{\kappa}$ of $P_{\kappa}$-points is equal to the intersection of all sets of the form $S_{\kappa} \mid(\bar{U} \backslash U)$, where $U$ is an open set, $\tau(U)<\kappa$. The number of such sets $U$ is $\kappa^{\kappa}=\kappa$ and thus $P_{\kappa}$ is a dense subset of $S_{\kappa}$. It can 
easily be seen that $\left|P_{\kappa}\right|=2^{\kappa}$. Since $S_{\kappa}$ is compact, $P_{\kappa} \neq S_{\kappa}$, and since under a homeomorphism a $P_{\kappa}$-point must be mapped to a $P_{\kappa}$-point, $S_{\kappa}$ is not point-homogeneous.

3.6. CoRollary. Let $U$ be an open set of $S_{\kappa}, 1<\tau(U)<\kappa$, and p a $P_{\kappa}$-point. Then the spaces $S_{\kappa}-\bar{U}, S_{\kappa}-\{p\}$ are each homeomorphic to $\Omega_{\kappa}$ (defined in 3.4).

For the proof use 1.5 and 3.4. See also 2.2 in [CN], for the case $\kappa=\omega_{1}$.

3.7. THEOREM. If $U$ is an open subset of $S_{\kappa}, \tau(U)=\kappa$, then there is a two-valued continuous function on $U$ which cannot be extended to $S_{\kappa}$.

The proof follows in the same way as in [FG]; see also $\left[\mathbf{N}_{2}\right]$ for a generalization of [FG] in a different direction.

Let $\Lambda_{\kappa}$ be the set $2^{\kappa}$ of all $\{0,1\}$-sequences indexed by $\kappa$, ordered lexicographically and endowed with the order topology. Since $\Lambda_{\kappa}$ is Dedekind complete, with a first and last element, it is compact in its order topology. For any space $S$, we let $P_{x}(S)$ denote the set of $P_{x}$-points in $S$ (thus $P_{\kappa}=P_{x}\left(S_{x}\right)$ ) and we let $\pi_{x}(S)$ denote the set $S$, with a base for the topology consisting of the family of all intersections of less than $\kappa$ open sets. Every element of $\pi_{\kappa}(S)$ is a $P_{\kappa}$-point. We state here the extension of the results of Parovičenko [P], omitting the similar proofs (cf. [CN]).

3.8. THEOREM. The following spaces are all homeomorphic: $\pi_{\kappa}\left(\Lambda_{\kappa}\right), P_{\kappa}\left(\Lambda_{\kappa}\right)$, $P_{\kappa}, \pi_{\kappa}\left(S_{\kappa}\right)$. In particular, $S_{\kappa}$ contains densely an $\eta_{\kappa}$-set of cardinality $\kappa$.

We conclude with a problem. The space $S_{\kappa}$ has not been constructed in an explicit way, and its topological properties were consequences of the fact that $\mathscr{B}_{\kappa}$ is $\kappa$-saturated. For $\kappa=\omega_{1}$, the natural homeomorph of $S_{\kappa}$ is $\beta \omega \mid \omega$. (As it has been noted above, the explicit statement of this fact is due to Keisler [K].) Theorem 3.8 suggests the construction of $S_{\kappa}$ as the compactification of an $\eta_{\kappa}$-set of cardinality $\kappa$. We have not been able to carry out such a construction. We note that for $\kappa>\omega$ the two "natural" compactifications of an $\eta_{\kappa}$-set of cardinality $\kappa$, namely the StoneCech compactification and the ordered compactification (which can be identified with the space $\Lambda_{\kappa}$ minus its set of upper points in the terminology of Gillman and Jerison [GJ]) are not homeomorphic to $S_{\kappa}$. The Stone-Cech compactification has cardinality $2^{2^{x}}$, while $S_{\kappa}$ has cardinality $2^{x}$. The ordered compactification cannot be even an $F$-space in the terminology of [GJ], since otherwise it would have to be a $P$-space (see Problem 50 in [GJ]), and that is not compatible with compactness.

4. We conclude with some remarks on the closed extremally disconnected subspaces of $S_{\kappa}$. A space is extremally disconnected if every open set has an open closure. The injective objects in the category of Boolean algebras are precisely the complete Boolean algebras (Sikorski $\left[\mathbf{S}_{2}\right]$ ). A more general result (Gleason [G]) identifies the projective objects in the category of compact spaces with the extremally disconnected spaces. Incidentally, we remark that Sikorski's theorem, Stone's duality for Boolean algebras, together with Lemmas 2.1, 2.2 actually imply Gleason's theorem. 
4.1. THEOREM. (a) If $E$ is an extremally disconnected compact space, with at most $\kappa$ open-and-closed sets, then $E$ can be embedded as a retract in $S_{\kappa}$.

(b) If $E$ is homeomorphic to a retract of $S_{k}$, with less than $\kappa$ open-and-closed sets, then $E$ is extremally disconnected.

Proof. (a) There is a continuous epimorphism $f: S_{x} \rightarrow E$. Let $E^{\prime}$ be a closed subset of $S_{\kappa}$, such that $f \mid E^{\prime}$ is irreducible, i.e. $f\left(E^{\prime}\right)=E$, and $f(F) \neq E$ for every proper closed subset of $E^{\prime}$. By Gleason's Lemma $[\mathbf{G}], f \mid E^{\prime}$ is a homeomorphism. It is easy now to see that $E^{\prime}$ is a retract of $S\left(\right.$ cf. $\left.\left[\mathrm{N}_{1}\right]\right)$.

(b) Using 2.1, 2.2, 2.3 we can prove that $S_{x}$ is projective in the category of all compact spaces of weight less than $\kappa$. A retract $E$ of a projective object is projective. If in addition $E$ has less than $\kappa$ open-and-closed sets, then it is easy to prove that $E$ is projective in the category of all compact spaces (and continuous maps), and thus by Gleason's Theorem, $E$ is extremally disconnected.

Note that 4.1 does not claim that if $E$ is a closed subset of $S_{x}, E$ extremally disconnected (even with less than $\kappa$ open-and-closed sets), then $E$ is a retract of $S_{\kappa}$. This must be left as a question.

\section{REFERENCES}

[B $\mathbf{B}_{1}$ ] Heinz Bachmann, Transfinite Zahlen, Zweite Auflage, Springer-Verlag, Berlin, 1967.

$\left[\mathrm{B}_{2}\right]$ D. D. Booth, Some consistency questions in topology, Notices Amer. Math. Soc. 15 (1968), 641.

[CN] W. W. Comfort and S. Negrepontis, Homeomorphs of three subspaces of $\beta N \backslash N$, Math. Z. 107 (1968), 53-58.

[DY] Ph. Dwinger and F. M. Yaqub, Generalized free products of Boolean algebras with an amalgamated subalgebra, Indag. Math. 25 (1963), 225-231.

[E] A. S. Esenin-Vol'pin, On the existence of a universal bicompactum of arbitrary weight, Dokl. Akad. Nauk SSSR 68 (1949), 649-652.

[F] Zdeněk Frolik, Sums of ultrafilters, Bull. Amer. Math. Soc. 73 (1967), 87-91.

[FG] N. J. Fine and L. Gillman, Extension of continuous functions in $\beta N$, Bull. Amer. Math. Soc. 66 (1960), 376-381.

[G] Andrew M. Gleason, Projective topological spaces, Illinois J. Math. 2 (1958), 482-489.

[GJ] Leonard Gillman and Meyer Jerison, Rings of continuous functions, Van Nostrand, Princeton, N.J., 1960.

[HR] Kenneth Hoffman and Arlan Ramsay, Algebras of bounded sequences, Pacific J. Math. 15 (1965), 1239-1248.

[ $\mathbf{J}_{1}$ ] B. Jónsson, Universal relational systems, Math. Scand. 4 (1956), 193-208.

$\left[\mathrm{J}_{2}\right] \longrightarrow$, Homogeneous universal relational systems, Math. Scand. 8 (1960), 137-142.

[K] H. Jerome Keisler, Universal homogeneous Boolean algebras, Michigan Math. J. 13 (1966), 129-132.

[MV] Michael Morley and Robert Vaught, Homogeneous universal models, Math. Scand. 11 (1962), 37-57.

[N] Stelios Negrepontis, On a theorem by Hoffman and Ramsay, Pacific J. Math. 20 (1967), 281-282.

$\left[\mathrm{N}_{2}\right]-$ Extension of continuous functions in $\beta D$, Indag. Math. 30 (1968), 393-400. 
[P] I. I. Parovičenko, A universal bicompact of weight $\$$, Dokl. Akad. Nauk SSSR 150 (1963), 36-39.

[R $\mathbf{R}_{1}$ ] Mary Ellen Rudin, “Types of ultrafilters," in Topology Seminar, Wisconsin, 1965, Ann. of Math. Studies No. 60, Princeton Univ. Press, Princeton, N. J., 1966, pp. 147-151.

$\left[\mathbf{R}_{2}\right]$ Walter Rudin, Homogeneity problems in the theory of Cech compactifications, Duke J. Math. 23 (1956), 409-419.

[S1] Waclaw Sierpiński, Sur les espaces métriques universels, Fund. Math. 33 (1945), 123-136.

[S $\mathbf{S}_{2}$ ] Roman Sikorski, Boolean algebras, 2nd ed., Springer-Verlag, Berlin, 1964.

\author{
MCGILl UNIVERSTTY, \\ MONTRÉal, QúEBeC
}

